\title{
Mortes de mulheres internadas para parto e por aborto e de seus conceptos em maternidades públicas
}

\author{
Deaths of women hospitalized for childbirth and abortion, and of \\ their concept, in maternity wards of Brazilian public hospitals
}

Pauline Lorena Kale ${ }^{1}$

Maria Helena Prado de Mello Jorge ${ }^{2}$

Sandra Costa Fonseca ${ }^{3}$

Angela Maria Cascão ${ }^{4}$

Kátia Silveira da Silva ${ }^{5}$

Ana Cristina Reis ${ }^{6}$

Mauro Tomoyuki Taniguchi ${ }^{7}$

${ }^{1}$ Departamento de Medicina Preventiva, Faculdade de Medicina, Universidade Federal do Rio de Janeiro. Av. Brigadeiro Trompowsky s/n, Ilha do Fundão. 21949900 Rio de Janeiro RJ Brasil.

pkale@iesc.ufri.br

${ }^{2}$ Faculdade de Saúde Pública, Universidade de São Paulo. São Paulo SP Brasil.

${ }^{3}$ Instituto de Saúde Coletiva, Universidade Federal Fluminense. Niterói RJ Brasil.

${ }^{4}$ Assessoria de Dados Vitais, Secretaria de Saúde do Estado do Rio de Janeiro. Rio de Janeiro RJ Brasil. ${ }^{5}$ Instituto Nacional de

Saúde da Mulher, da Criança e do Adolescente Fernandes Figueira, Fundação Oswaldo Cruz (FioCruz). Rio de Janeiro RJ Brasil.

${ }^{6}$ Escola Politécnica de Saúde Joaquim Venâncio, Fiocruz. Rio de Janeiro RJ Brasil.

${ }^{7}$ Programa de Aprimoramento das Informações de Mortalidade no Município de São Paulo, Secretaria Municipal de Saúde. São Paulo SP Brasil.
Abstract The aim of this cross-sectional hospital-based study of 7,845 pregnancies was to analyze deaths of women hospitalized for childbirth and abortion, and fetal and neonatal deaths, in public hospitals in the cities of São Paulo, Rio de Janeiro and Niteroi (RJ), Brazil, in 2011. Outcomes of the pregnancies were: one maternal death, 498 abortions, 65 fetal deaths, 44 neonatal deaths and 7,291 infant survivors. Data were collected through interviews, medical records and the women's pregnancy records, and from the Mortality Information System (SIM). The study population was described and kappa coefficients of causes of death (from the SIM, and certified by research) and mortality health indicators were estimated. The maternal mortality ratio was 13.6 per 100,000 live births (LB), the fetal death rate was $8.8 \%$ births and the neonatal mortality rate was $6.0 \%$ LB. The drug most used to induce abortion was Misoprostol. The main causes of fetal and neonatal deaths were respiratory disorders and maternal factors. Congenital syphilis, diabetes and fetal death of unspecified cause were under-reported in the SIM. Kappa coefficients by chapter were 0.70 (neonatal deaths) and 0.54 (stillbirths). Good quality care in reproductive planning, prenatal care, during labor and at birth will result in prevention of deaths.

Key words Abortion, Fetal death, Neonatal mortality, Maternal mortality, Cause of death
Resumo O objetivo deste estudo foi analisar mortes de mulheres internadas para parto e por aborto, e de seus conceptos - fetais e neonatais - em maternidades públicas nas cidades de São Paulo, Rio de Janeiro e Niterói (RJ), em 2011. Estudo seccional de base hospitalar. Participaram 7.845 mulheres resultando em um óbito materno, 498 abortos, 65 óbitos fetais, 44 óbitos neonatais e 7.291 sobreviventes infantis. Dados foram obtidos por meio de entrevista, consulta ao prontuário, cartão da gestante e no Sistema de Informações sobre Mortalidade (SIM). Foi descrita a população de estudo e estimados a concordância da causa básica (SIM e certificada pela pesquisa) e os indicadores de mortalidade. A mortalidade materna foi 13,6 por cem mil nascidos vivos (NV), fetal 8,8\%o nascimentos e neonatal 6,0\% NV. Misoprostol foi o medicamento mais utilizado no aborto provocado. Transtornos respiratórios $e$ fatores maternos foram as principais causas entre óbitos fetais e neonatais. Sífilis congênita, diabetes $e$ causas de morte fetal não especificada foram subdeclaradas no SIM. Os coeficientes kappa por capitulo foram 0,70 (neonatais) e 0,54 (natimortos). A assistência de boa qualidade no planejamento reprodutivo, pré-natal, durante o parto e nascimento resultará na prevenção das mortes.

Palavras-chave Aborto, Morte fetal, Mortalidade neonatal, Mortalidade materna, Causas de morte 


\section{Introdução}

Mulheres e seus conceptos representam um grupo de maior vulnerabilidade, expressa pelo risco de adoecer e morrer ${ }^{1}$, cujos determinantes biológicos são potencializados por características socioeconômicas e assistenciais².

Morte materna é um evento raro, trágico e evitável ${ }^{3}$. Em 2013, apesar dos avanços na saúde materno-infantil no país, a razão de mortalidade materna foi 58,1 por 100.000 nascidos vivos ${ }^{4}$, não alcançando a redução de $75 \%$, quinta Meta dos Objetivos do Desenvolvimento do Milênio ${ }^{5}$.

Quanto ao concepto, o risco de morte é maior no início da gravidez quando essa ainda não foi sequer reconhecida ou diagnosticada ${ }^{6}$. Estas perdas da gravidez, frequentemente relatadas como irregularidades menstruais, não costumam ser registradas ${ }^{6}$.

A magnitude dos abortos também é imprecisa, mesmo em países onde é legalizado ${ }^{7}$. Em 2010, a Pesquisa Nacional de Aborto estimou que $15 \%$ das mulheres brasileiras no final da vida reprodutiva já tinham feito aborto, necessitando internação em $50 \%$ dos casos ${ }^{8}$. Apesar da redução de $26 \%$ das estimativas nacionais indiretas de abortos induzidos em mulheres em idade fértil de 1995 a 2013, sua magnitude é preocupante9.

O que define se as perdas fetais são abortos ou óbitos fetais são pontos de corte relativos ao peso ao nascer, idade gestacional ou comprimento do concepto $^{10}$. O Brasil adota a definição da Classificação Estatística Internacional de Doenças e Problemas Relacionados à Saúde $10^{a}$ Revisão (CID), que propõe como pontos de corte para óbito fetal: peso ao nascer: 500 gramas; idade gestacional: $22^{\text {a }}$ semanas e comprimento de $25 \mathrm{~cm}^{10}$. Lawn et al. ${ }^{11}$ recomendam que a definição de óbito fetal, para comparações internacionais, use idade gestacional (28 ou mais semanas) e propõem essa mudança na $11^{\text {a }}$ Revisão da CID. A mortalidade fetal é pouco compreendida e estudada, não foi incluída entre os Objetivos de Desenvolvimento do Milênio e encontrava-se praticamente invisível nas agendas políticas mundiais ${ }^{11}$. No entanto, as taxas continuam elevadas, principalmente nos países de baixa e média rendas; estimando-se a taxa média nestes países em 28,9 óbitos fetais por mil nascimentos variando de 13,6, na Argentina a 56,5, no Paquistão ${ }^{12}$. Em 2014, a $67^{a}$ Assembleia Mundial de Saúde estabeleceu como meta a redução da mortalidade fetal para 12 ou menos por mil nascimentos em $2030^{11}$.

Para os nascidos vivos, o risco de morte infantil é maior imediatamente após a transição para a vida extrauterina e decresce marcadamente durante a primeira semana de vida ${ }^{5,6,13}$. Considerando-se a similaridade das causas de mortes e dos fatores de risco entre os óbitos fetais e neonatais precoces, não é esperada uma variação na qualidade do preenchimento da Declaração de Óbito. Entretanto, erros sistemáticos geram uma pior qualidade da informação para óbitos fetais ${ }^{14}$. Incompletude, baixa confiabilidade e validade das causas de morte para óbitos neonatais e fetais, principalmente, foram relatadas em estudos nacionais ${ }^{15-18}$.

Se por um lado avanços tecnológicos da assistência à gravidez e ao período neonatal trouxeram resultados positivos para a saúde perinatal, por outro acentuaram as desigualdades onde o acesso à tecnologia não é universal e equitativo ${ }^{19}$. Desigualdades de assistência à saúde e sociodemográficas são fatores de risco comuns a abortos induzidos $^{20}$, óbitos fetais ${ }^{15}$, óbitos neonatais ${ }^{13} \mathrm{e}$ óbitos maternos ${ }^{3}$.

A hipertensão e a hemorragia têm sido as causas mais frequentes de morte materna no país e a análise das causas dos óbitos neonatais e fetais mostra as morbidades maternas como causa crescente $^{4,18}$, mostrando a inter-relação entre estes óbitos e as intervenções necessárias para sua redução. Durante o pré-natal, o rastreio e tratamento de morbidades como sífilis, diabetes, hipertensão arterial $^{21}$, bem como a habilidade para detectar e manusear o trabalho de parto obstruído e a redução da medicalização no momento do parto podem prevenir mortes de mães e seus conceptos ${ }^{22}$.

O objetivo deste estudo foi analisar, em maternidades do Sistema Único de Saúde, os óbitos maternos, abortos, óbitos fetais e neonatais, nas cidades de São Paulo, Rio de Janeiro e Niterói (RJ) em 2011, com ênfase nas causas de mortes.

\section{Metodologia}

Foi realizado um estudo seccional em maternidades com maior número de nascimentos do Sistema Único de Saúde (SUS):quatro na cidade de São Paulo, SP (centro-SP), uma em Niterói e outra no Rio de Janeiro, RJ (centro-RJ) - em três meses do segundo semestre de 2011. As mortes hospitalares ocorridas durante a gestação, no parto e até a alta foram identificadas durante o trabalho de campo e, posteriormente, por relacionamento probabilístico das bases de dados da pesquisa com o Sistema de Informações sobre Mortalidade (SIM), em São Paulo, pelo Programa de Aprimoramento 
das Informações de Mortalidade - PRO-AIM, da Prefeitura do Município de São Paulo e no Rio de Janeiro e Niterói, pela Secretaria de Estado de Saúde do Rio de Janeiro. Foi utilizado o programa computacional RecLink $3^{23}$. As perdas do estudo foram inferiores a $5 \%$.

Foram realizadas entrevistas às mulheres após 12 horas do parto e cinco horas após o aborto, consultas aos prontuários hospitalares, cartão da gestante e registro de sala de parto, por enfermeiros e estudantes da área da saúde previamente treinados.

\section{População de estudo}

O fluxograma da população de estudo relativo às possíveis situações das mulheres e de seus conceptos está representado na Figura 1.
Foram entrevistadas 7.845 mulheres (centros SP: 5.955 e RJ: 1.890). No centro SP ocorreram quatro óbitos de mulheres, um materno após transferência e três após a alta hospitalar, ocorridos no puerpério tardio por causas não relacionadas ao ciclo gravídico-puerperal, não computados como morte materna. Estes óbitos foram investigados pelo Comitê de Morte Materna, Fetal e Infantil do Município (CMMIF) de São Paulo. Não ocorreu óbito de mulher no centro RJ.

Foram analisadas as perdas fetais e óbitos de nascidos vivos segundo o momento de ocorrência: abortos e óbitos fetais $(<22$ e $\geq 22$ semanas de gestação, respectivamente) e neonatais (0-27 dias) ${ }^{10}$. Apesar da definição de óbitos fetais (morte de um produto da concepção, antes da expulsão ou da extração completa do corpo da mãe, independentemente da duração da gravidez) $)^{10}$,

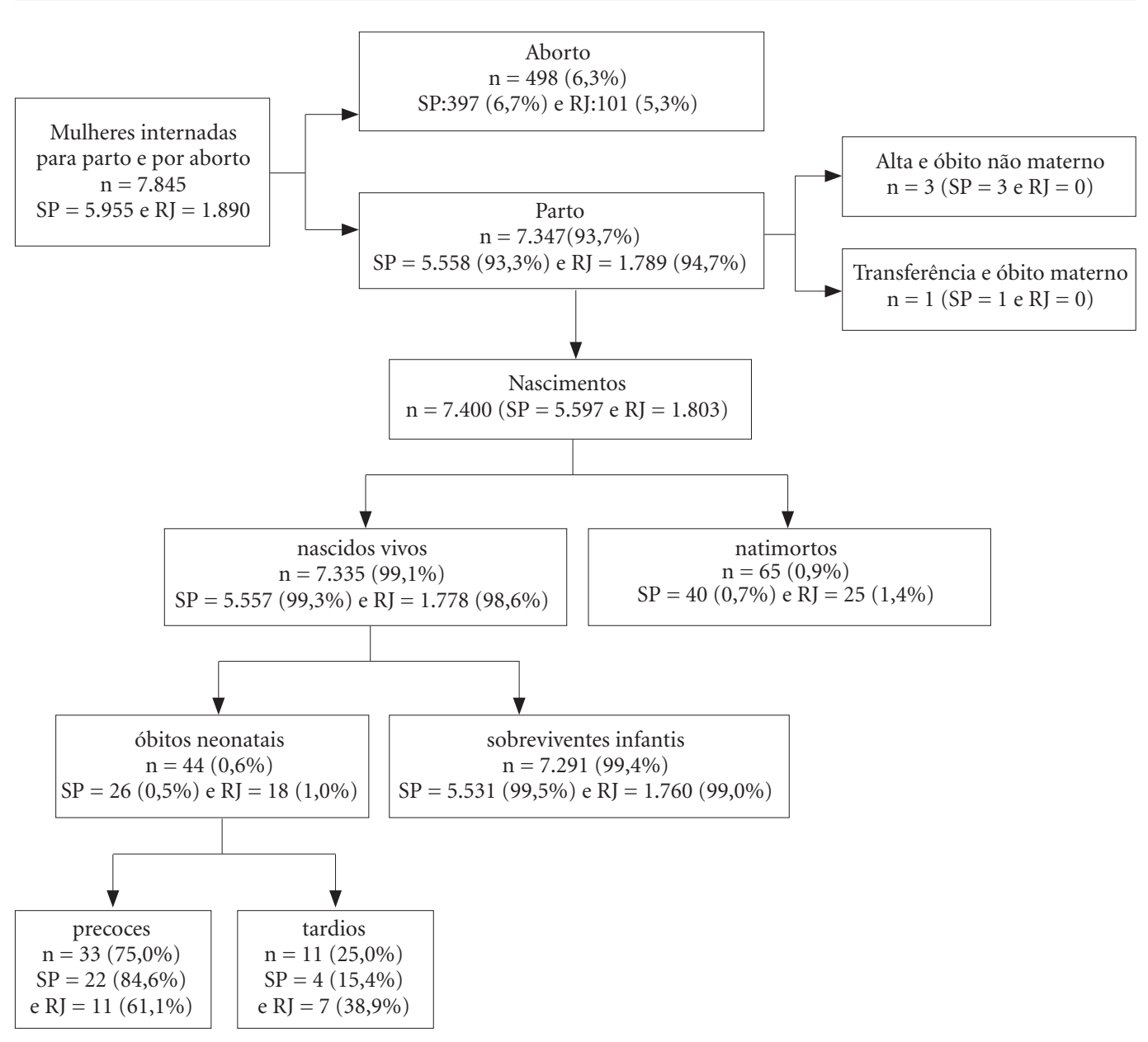

Figura 1. Fluxograma das mulheres e de seus conceptos, em maternidades públicas das cidades São Paulo (centro São Paulo - SP), Rio de Janeiro e Niterói (centro Rio de Janeiro - RJ), 2011. 
adotamos óbito fetal para representar o componente fetal da mortalidade perinata ${ }^{10}$, para fins de comparação com outros estudos. Um aborto foi reclassificado como natimorto. A partir das fontes oficiais foi possível identificar os óbitos infantis ocorridos após a pesquisa e, portanto, os sobreviventes ao período infantil (364 dias completos de vida). Não foram analisados 26 óbitos infantis ocorridos após a alta hospitalar devido à impossibilidade de acesso ao prontuário médico para certificação de uma nova Declaração de Óbito. Estes óbitos foram excluídos da análise do grupo de sobreviventes. Do total de 7.898 conceptos, analisaram-se 563 perdas fetais (498 abortos e 65 óbitos fetais), 44 óbitos neonatais (33 precoces e 11 tardios) e 7.291 sobreviventes infantis.

As frequências de mortes dos conceptos foram apresentadas segundo o momento da morte. Abortos foram descritos segundo o tipo (sem expulsão do feto; espontâneo; induzido e previsto em lei ou por ordem judicial; provocado) e o meio utilizado para abortos provocados. Óbitos fetais e neonatais informados pelo SIM foram descritos segundo a realização de investigação.

Conceptos foram descritos segundo centro de ocorrência do parto ou aborto (centro São Paulo; centro Rio de Janeiro) e características sociodemográficas maternas, como faixa etária $(<20 ; 20-34 ; \geq 35$ anos), escolaridade $(<8$; $\geq 8$ anos de estudo) e cor da pele autorreferida (branca; parda; negra; outras), características da história reprodutiva e da gestação, como número de partos anteriores (nenhum, 1-2 e 3 ou mais), aborto anterior (sim; não e não se aplica), natimortalidade anterior (sim; não e não se aplica), e, aferidas de forma binária (sim; não), morbidades autorreferidas durante a gestação (pressão alta, hemorragia e sífilis); comportamento de risco durante a gestação (uso de fumo, álcool, droga ilícita), presença de companheiro e realização do pré-natal. Adicionalmente, o óbito materno foi descrito segundo nacionalidade, assistência obstétrica prestada, causa de morte e desfecho do produto da gestação. Peso ao nascer (PN em gramas) e idade gestacional (IG em semanas) foram descritos para os subgrupos da população de estudo, exceto aborto, a partir dos indicadores: proporção de extremo baixo $\mathrm{PN}(<1.000 \mathrm{~g})$, de muito baixo $\mathrm{PN}(<1.500 \mathrm{~g})$, de baixo $\mathrm{PN}(<$ $2.500 \mathrm{~g})$ e de muito pré-termo ( $<32$ semanas) e pré-termo ( $<37$ semanas). Foram excluídos da análise de idade gestacional (IG) e peso ao nascer $(\mathrm{PN})$, registros com inconsistência entre estas informações ${ }^{24}$ : IG < 22 semanas e PN > 600g; IG de 22-27 semanas e PN > 1.500g; IG de 37-41 se- manas ou IG $\geq 42$ semanas e $\mathrm{PN}<1500$ g ou $>$ 5500 g. Somente para os óbitos neonatais e sobreviventes foram descritas as frequências de nascidos vivos (NV) com Apgar no quinto minuto $<7$.

Óbitos fetais e neonatais foram classificados por causas específicas (categorias de três e quatro caracteres), agrupamentos e capítulos da $\mathrm{CID}^{10}$. $\mathrm{Na}$ presença de investigação dos óbitos fetais e neonatais, as causas de morte referem-se àquelas pós-investigação registradas no SIM. Adicionalmente, os pesquisadores desconhecendo tais informações preencheram um novo atestado de óbito (São Paulo pelo coordenador geral da pesquisa e Estado do Rio de Janeiro, pela terceira autora do artigo), codificaram as causas de mortes e selecionaram a causa básica (quarta autora do artigo).

As análises das causas de morte foram realizadas separadamente para óbitos fetais e óbitos neonatais e por fonte certificadora (SIM e pesquisa). A causa básica de morte (CB) foi classificada segundo capítulo e agrupamento da CID e por causas específicas (categorias de três e quatro caracteres) apenas para os principais agrupamentos. As mudanças na frequência de óbitos segundo capítulo e agrupamento informado pelo SIM e certificada pela pesquisa por momento do óbito (fetal/neonatal) foram calculadas: valores positivos expressam aumento e negativos, diminuição. Foi descrita e contabilizada a frequência de diagnósticos além da $\mathrm{CB}$, independentemente de sua localização no Atestado de Óbito (mesma linha ou não da parte I ou na parte II) e analisada a confiabilidade da CB informada pelo SIM e certificada pelos pesquisadores.

\section{Análises Estatísticas}

Foram calculados indicadores de mortalidade hospitalar: razão de mortalidade materna por $100.000 \mathrm{NV}$, risco de morte fetal, óbitos fetais por 1.000 nascimentos e o risco de morte neonatal por $1.000 \mathrm{NV}^{10}$.

As distribuições, absoluta e percentual dos conceptos segundo características socioeconômicas, demográficas, história reprodutiva, da gestação e do parto foram descritas. Para testar a hipótese de homogeneidade de proporções foram realizados teste do Qui-quadrado de Pearson ou teste exato de Fisher. Para variáveis categóricas ordinais utilizou-se o teste do Qui-quadrado de tendência linear.

A distribuição e o posto de frequência da $\mathrm{CB}$ por capítulo e agrupamento da CID e por causas específicas (três e quatro caracteres) apenas para 
os principais agrupamentos foram descritas. Foram calculadas a média e a frequência absoluta de diagnósticos além da CB declarados (SIM e pesquisa). Foi realizado o teste de McNemar para investigar diferença entre as proporções de óbitos por causas por capítulo e agrupamento da CID, informada pelo SIM e pelos pesquisadores. Para a avaliação da concordância da CB (variáveis dicotômicas da causa específica - sim/não - informada pelo SIM e pela pesquisa) calculou-se a concordância percentual (<25\% - ruim; de 25\% a $49 \%$ - regular; de $50 \%$ a $74 \%$ - boa e $\geq 75 \%$ muito boa $)^{25}$ e o coeficiente kappa $(<0,00-$ pobre; 0,00-0,20 - superficial; 0,21-0,40 - razoável; 0,41-0,60 - moderada; 0,61-0,80 - substancial; $0,81-1,00$ - quase perfeita $)^{26}$.

\section{Considerações éticas}

Este estudo foi aprovado pelo Comitê de Ética em Pesquisa do Instituto de Estudos em Saúde Coletiva da Universidade Federal do Rio de Janeiro, da Secretaria de Saúde e Defesa Civil do Município do Rio de Janeiro, Centros de Estudos de cada Maternidade do Estado do Rio de Janeiro, pelo Comitê de Ética em Pesquisa da Faculdade de Saúde Pública da Universidade de São Paulo e hospitais participantes de São Paulo. Foi solicitado o consentimento informado a todas as gestantes elegíveis ou às respectivas responsáveis quando as mesmas eram menores de 18 anos.

\section{Resultados}

A razão de mortalidade materna hospitalar foi 13,6 por $100.000 \mathrm{NV}$, e o risco de morte fetal foi 8,8 por 1.000 nascimentos (centros RJ: 13,9\%o, e SP: 7,1\%o). A mortalidade neonatal foi 6,0 por 1.000 NV (centros RJ: 10,1\%o e SP: 4,7\%o).

\section{População de estudo}

O único óbito materno era de uma mulher residente no município de São Paulo, natural da Bolívia, de 34 anos, cor amarela e baixa escolaridade. Ela não vivia com companheiro, era do lar, teve cinco gravidezes anteriores (um aborto e quatro NV), e a atual resultou em óbito fetal (560 gramas e 23 semanas). Não fez o pré-natal e não referiu morbidade e comportamento de risco durante a gestação. $\mathrm{O}$ óbito ocorreu no puerpério imediato após transferência para hospital de maior complexidade. A CB de morte foi outras hemorragias do pós-parto imediato (código da CID: O72) e constava como causa associada tuberculose pulmonar (código da CID: A162).

Entre os 7.898 conceptos ocorreram 498 abortos -29,1\% sem expulsão do embrião, 64,5\% espontâneos, $0,4 \%$ induzidos, previstos por lei ou ação judicial, 3,2\% provocados e 2,8\%, ignorados. O meio de abortamento provocado mais utilizado foi o misoprostol. A proporção de aborto provocado no centro RJ $(11,9 \%)$ foi cerca de 12 vezes maior do que o centro SP (1\%), enquanto que o aborto espontâneo foi 30\% superior no centro SP $(68,1 \%)$ em relação ao centro RJ (50,5\%). Entre os nascimentos, 65 eram óbitos fetais e $7.335 \mathrm{NV}$ ocorrendo 44 óbitos neonatais hospitalares.

A Tabela 1 apresenta a distribuição dos conceptos segundo características sociodemográficas, da história reprodutiva e gestação, comportamento de risco, assistência à saúde e características dos recém-nascidos.

Conceptos foram heterogêneos em todas as características sociodemográficas maternas ( $p$ valor $<0,05)$. Destacaram-se nas faixas etárias extremas, $34 \%$ de adolescentes entre os óbitos fetais e $20 \%$ com 35 anos e mais entre os abortos. As proporções de mulheres de cor da pele preta entre as mortes fetal e neonatal foram superiores àquelas dos sobreviventes (13,5\%), alcançando $30,2 \%$ entre os óbitos neonatais. Evidenciou-se um percentual maior de baixa escolaridade entre mães dos óbitos fetais (32,3\%). Prevaleceu presença do companheiro entre óbitos neonatais e sobreviventes e ausência deste entre abortos e óbitos fetais.

Exceto por natimortalidade prévia, todas as demais características reprodutivas diferiram segundo o tipo de desfecho ocorrido ( $p$ valor $<$ $0,0001)$. Entre as mulheres que tiveram aborto, $11,1 \%$ tinham história de quatro partos ou mais. Aborto prévio foi relatado com mais frequência entre os subgrupos de morte quando comparados ao subgrupo de sobrevivência.

A hipertensão arterial e a sífilis materna foram mais frequentes exceto entre os abortos, quando prevaleceu hemorragia. Tabagismo, uso de álcool e de drogas foram mais comuns entre as mães dos óbitos neonatais. Nesse grupo, nenhuma mãe declarou ter sofrido violência durante a gestação. Dentre as morbidades e comportamentos de risco, somente hipertensão arterial e uso de álcool apresentaram significância estatística $(\mathrm{p}<0,05)$. Não ter realizado o pré-natal foi mais frequente entre os casos de aborto (63\%). Baixo peso ao nascer e prematuridade foram mais frequentes entre óbitos fetais e neonatais ( $\mathrm{p}<$ 0,0001). 
Tabela 1. População de estudo segundo desfecho da gestação e algumas características maternas e dos conceptos nas cidades Rio de Janeiro, Niterói (centro RJ) e São Paulo (centro SP), 2011.

\begin{tabular}{|c|c|c|c|c|c|c|c|c|c|}
\hline \multirow[t]{2}{*}{ Características } & \multicolumn{2}{|c|}{ Aborto } & \multicolumn{2}{|c|}{ Óbito fetal $^{a}$} & \multicolumn{2}{|c|}{$\begin{array}{c}\text { Óbito } \\
\text { neonatal }\end{array}$} & \multicolumn{2}{|c|}{$\begin{array}{c}\text { Sobreviventes } \\
\text { Infantis }\end{array}$} & \multirow[t]{2}{*}{ p-valor } \\
\hline & $\mathrm{n}=498$ & $\%$ & $\mathrm{n}=65$ & $\%$ & $\mathrm{n}=44$ & $\%$ & $\mathrm{n}=7291$ & $\%$ & \\
\hline \multicolumn{10}{|l|}{ Sociodemográficas maternas } \\
\hline Centro do parto ou aborto & & & & & & & & & $<0,0001$ \\
\hline São Paulo & 397 & 79,7 & 40 & 61,5 & 26 & 59,1 & 5531 & 75,9 & \\
\hline Rio de Janeiro & 101 & 20,3 & 25 & 38,5 & 18 & 40,9 & 1760 & 24,1 & \\
\hline Faixa etária & & & & & & & & & $<0,0001$ \\
\hline $10-19$ & 75 & 15,1 & 22 & 33,8 & 8 & 18,2 & 1618 & 22,2 & \\
\hline $20-34$ & 322 & 64,7 & 34 & 52,3 & 33 & 75,0 & 5022 & 68,9 & \\
\hline 35 e mais & 101 & 20,3 & 9 & 13,8 & 3 & 6,8 & 651 & 8,9 & \\
\hline Cor da pele & & & & & & & & & 0,001 \\
\hline branca & 197 & 40,9 & 19 & 30,2 & 17 & 39,5 & 2630 & 36,9 & \\
\hline parda & 188 & 39,0 & 33 & 52,4 & 12 & 27,9 & 3354 & 47,1 & \\
\hline preta & 86 & 17,8 & 10 & 15,9 & 13 & 30,2 & 959 & 13,5 & \\
\hline outras & 11 & 2,3 & 1 & 1,6 & 1 & 2,3 & 182 & 2,6 & \\
\hline Escolaridade & & & & & & & & & 0,047 \\
\hline$<8$ anos & 85 & 17,5 & 20 & 32,3 & 7 & 16,7 & 1393 & 19,7 & \\
\hline$>=8$ anos & 402 & 82,5 & 42 & 67,7 & 35 & 83,3 & 5690 & 80,3 & \\
\hline Companheiro presente & 168 & 34,1 & 15 & 23,4 & 29 & 65,9 & 4709 & 65,3 & $<0,0001$ \\
\hline \multicolumn{10}{|l|}{ História reprodutiva } \\
\hline No de partos anterirores ${ }^{b}$ & & & & & & & & & 0,01 \\
\hline Nenhum & 126 & 31,9 & 24 & 36,9 & 18 & 40,9 & 3062 & 42,0 & \\
\hline $1-3$ & 225 & 57,0 & 36 & 55,4 & 22 & 50,0 & 3720 & 51,1 & \\
\hline 4 e mais & 44 & 11,1 & 5 & 7,7 & 4 & 9,1 & 501 & 6,9 & \\
\hline Nascidos mortos anteriores ${ }^{\mathrm{d}}$ & 10 & 2,0 & 2 & 3,2 & - & - & 120 & 1,7 & - \\
\hline Abortos anteriores & 124 & 25,0 & 12 & 19,0 & 10 & 22,7 & 1303 & 17,9 & 0,01 \\
\hline \multicolumn{10}{|l|}{ Da gestação } \\
\hline Referiu hipertensão arterial & 17 & 3,4 & 12 & 18,5 & 6 & 13,6 & 853 & 11,7 & $<0,0001$ \\
\hline Referiu hemorragia $^{\mathrm{d}}$ & 90 & 18,1 & 5 & 7,7 & 6 & 13,6 & 402 & 5,5 & - \\
\hline Referiu Sífilis ${ }^{\mathrm{d}}$ & 3 & 0,6 & 6 & 9,2 & 3 & 6,8 & 140 & 1,9 & - \\
\hline Usou tabaco & 85 & 17,3 & 8 & 12,5 & 9 & 20,5 & 1062 & 14,6 & 0,252 \\
\hline Usou de álcool & 73 & 14,8 & 10 & 15,6 & 13 & 30,2 & 972 & 13,4 & 0,01 \\
\hline Usou de drogas ilícitas ${ }^{\mathrm{d}}$ & 11 & 2,2 & - & - & 3 & 7,0 & 96 & 1,3 & - \\
\hline Sofreu violência ${ }^{\mathrm{d}}$ & 24 & 4,9 & 6 & 9,7 & - & - & 283 & 3,9 & - \\
\hline Não fez o pré-natal ${ }^{\mathrm{d}}$ & 315 & 63,3 & 6 & 9,2 & 7 & 16,3 & 110 & 1,5 & - \\
\hline \multicolumn{10}{|l|}{ Recém-nascidos } \\
\hline Com peso ao nascer $<1000 \mathrm{~g}^{\mathrm{d}}$ & $\mathrm{NA}$ & NA & 20 & 34,5 & 21 & 53,8 & 6 & 0,1 & - \\
\hline Com peso ao nascer $<1500 \mathrm{~g}^{\mathrm{d}}$ & NA & NA & 33 & 56,9 & 28 & 71,8 & 38 & 0,7 & - \\
\hline Com peso ao nascer $<2500 \mathrm{~g}$ & NA & NA & 45 & 77,6 & 32 & 82,1 & 463 & 8,0 & $<0,0001$ \\
\hline Com idade gestacional $<32 \mathrm{sem}^{\mathrm{d}}$ semanas & NA & NA & 33 & 56,9 & 27 & 69,2 & 127 & 2,2 & - \\
\hline Com idade gestacional $<37$ sem semanas & NA & NA & 46 & 79,3 & 30 & 76,9 & 657 & 11,4 & $<0,0001$ \\
\hline Com Apgar $5 \min <7^{c}$ & NA & NA & NA & NA & 21 & 47,7 & 6 & 0,1 & $<0,0001$ \\
\hline
\end{tabular}

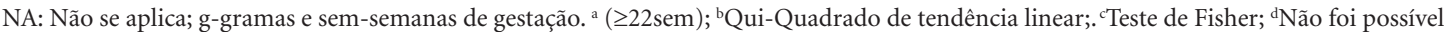
testar a significância estatística em função das baixas frequências. OBS: Foram excluídos da análise registros com informações ignoradas. Adicionalmente, para análise do peso ao nascer e idade gestacional foram excluídas as inconsistências entre as duas variáveis resultando em 58 natimortos, 39 óbitos neonatais e 5.775 sobreviventes infantis.

Considerando-se o momento da ocorrência da morte fetal e neonatal, observa-se a redução da frequência à medida que avança a idade gesta- cional e a idade cronológica (Gráfico 1). O declínio relativo do número de óbitos foi maior desde o início da gravidez (498 abortos) até a vigésima 


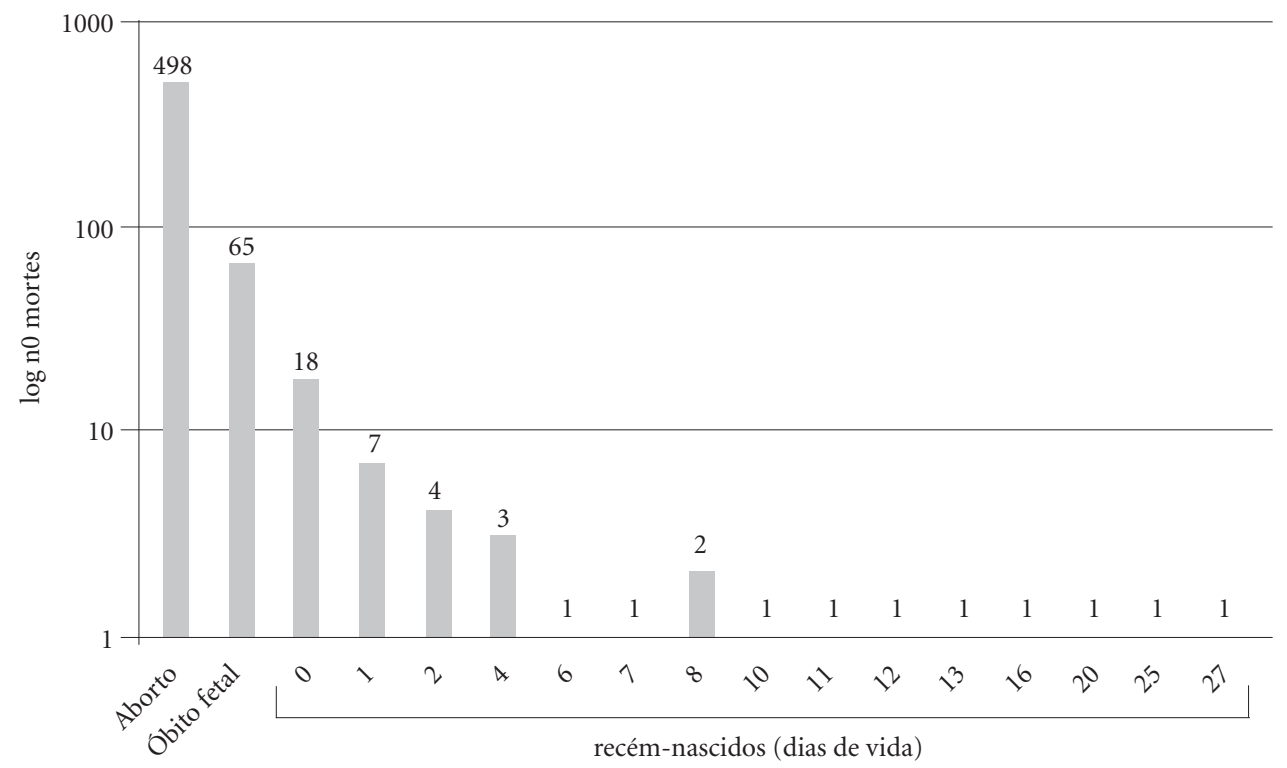

Gráfico 1. Distribuição das perdas fetais ${ }^{\star}$ e dos óbitos neonatais segundo o momento da morte em maternidades públicas das cidades Rio de Janeiro, Niterói e São Paulo, 2011.

${ }^{*}$ Perdas fetais: aborto e óbito fetal ( $<22$ e $\geq 22$ semanas de gestação, respectivamente).

segunda semana de gestação (65 óbitos fetais), cerca de $87 \%$ e desta até o primeiro dia de vida incompleto (18 óbitos neonatais), $72 \%$.

\section{Causas de morte dos conceptos}

A investigação de óbitos informados pelo SIM ocorreu em $83,1 \%$ dos óbitos fetais e $84,1 \%$ dos óbitos neonatais.As principais causas de mortes por capítulo da CID foram Causas Perinatais (XVI) seguidas de Malformações Congênitas (XVII) e Doenças Infecciosas e Parasitárias (I) tanto entre as informadas pelo SIM quanto as certificadas pelos pesquisadores. Um óbito neonatal foi certificado pelos pesquisadores como Causas Externas (XX). Para os óbitos fetais a certificação da pesquisa comparada à informação do SIM, aumenta a frequência de doenças infecciosas e parasitárias e malformações congênitas e diminui a frequência das causas perinatais; para os óbitos neonatais, verifica-se diminuição da frequência das malformações congênitas e aumento das demais.

A Tabela 2 apresenta a distribuição dos óbitos fetais e neonatais por $\mathrm{CB}$ e número de diagnós- ticos, além da CB segundo agrupamento da CID, informados pelo SIM e certificados pelos pesquisadores.

Entre os óbitos fetais, 78\% das CB informadas pelo SIM foram por Transtornos respiratórios e cardiovasculares específicos do período perinatal (P20-P29), exclusivamente por hipóxia intra-uterina não especificada (P209), e Fatores maternos e por complicações da gravidez, do trabalho de parto e parto (P00-P004), destacando-se neste agrupamento, doenças hipertensivas da gravidez (P000) $e$ outras formas de descolamento prematuro de placenta e hemorragia (P021). Quando certificado pela pesquisa passou a predominar o agrupamento Outros transtornos originados no período perinatal (P90-P97), exclusivamente devido às mortes fetais de causas não especificadas (P95), o correspondente às causas mal definidas para natimortos (Tabela 2). O número de diagnósticos, além da $\mathrm{CB}$, nos atestados de óbitos dos óbitos fetais variou entre zero e três; em cerca de 60\% (SIM) e $62,5 \%$ (pesquisadores) havia registro apenas da $\mathrm{CB}$, principalmente quando classificada no agrupamento Transtornos respiratórios e cardiovasculares (SIM) e Outros transtornos originados 
Tabela 2. Agrupamentos de causa básica $(\mathrm{CB})$ de natimortos e de óbitos neonatais, número de diagnósticos além da CB informados pelo Sistema de Informações sobre Mortalidade (SIM) e pela pesquisa, proporção de mudança da CB informada pelo SIM e certificada pela pesquisa, em maternidades das cidades de São Paulo, do Rio de Janeiro e Niterói, 2011.

\begin{tabular}{|c|c|c|c|c|c|c|c|c|c|c|c|c|c|c|c|}
\hline \multirow{3}{*}{$\begin{array}{l}\text { Causa Básica }(\mathrm{CB}) \text { por Agrupamentos } \\
\text { (Classificação Estatística } \\
\text { Internacional de Doenças e Problemas } \\
\text { Relacionados à Saúde - 10 } 0^{\mathrm{a}} \text { Revisão) }\end{array}$} & \multicolumn{7}{|c|}{ SIM } & \multicolumn{7}{|c|}{ Pesquisa } & \multirow{3}{*}{$\begin{array}{c}\text { Mudança } \\
\text { Causa } \\
\text { Básica } \\
\%\end{array}$} \\
\hline & \multirow{2}{*}{$\mathbf{n}^{0}$} & \multirow[t]{2}{*}{$\%$} & \multicolumn{5}{|c|}{$\begin{array}{l}\mathbf{n}^{0} \text { diagnósticos } \\
\text { além da } \mathrm{CB}\end{array}$} & \multirow{2}{*}{$\mathbf{n}^{0}$} & \multirow{2}{*}{$\%$} & \multicolumn{5}{|c|}{$\begin{array}{l}\mathbf{n}^{0} \text { diagnóticos } \\
\text { além da } \mathrm{CB}\end{array}$} & \\
\hline & & & 0 & 1 & 2 & 3 & $\geq 4$ & & & 0 & 1 & 2 & 3 & $\geq 4$ & \\
\hline \multicolumn{16}{|l|}{ Óbitos Fetais ${ }^{\mathrm{a}}$} \\
\hline $\begin{array}{l}\text { A50-A54: Infecções de transmissão } \\
\text { predominantemente sexual }\end{array}$ & 2 & 3,1 & - & 1 & - & 1 & - & 5 & 7,7 & 4 & 1 & - & - & - & 150,0 \\
\hline $\begin{array}{l}\text { P00-P04: Fatores maternos e } \\
\text { complicações (gravidez/trabalho de } \\
\text { parto/parto) }\end{array}$ & 20 & 31,3 & 1 & 14 & 4 & 1 & - & 21 & 32,3 & 4 & 9 & 5 & 3 & - & 5,0 \\
\hline $\begin{array}{l}\text { P05-P08: Transtornos relacionados } \\
\text { com duração da gestação e } \\
\text { crescimento fetal }\end{array}$ & - & - & - & - & - & - & - & 1 & 1,5 & 1 & - & - & - & - & - \\
\hline $\begin{array}{l}\text { P20-P29: Transtornos respiratórios/ } \\
\text { cardiovasculares específicos período } \\
\text { perinatal }\end{array}$ & 30 & 46,9 & 29 & 1 & - & - & - & 4 & 6,2 & 2 & 2 & - & - & - & $-86,7^{c}$ \\
\hline $\begin{array}{l}\text { P35-P39: Infecções específicas do } \\
\text { período perinatal }\end{array}$ & 1 & 1,6 & - & 1 & - & - & - & - & - & - & - & - & - & - & - \\
\hline $\begin{array}{l}\text { P70-P74: Transtornos endócrinos e } \\
\text { metabólicos transitórios e específicos }\end{array}$ & 1 & 1,6 & - & - & 1 & - & - & 5 & 7,7 & 2 & 1 & 1 & 0 & - & 300,0 \\
\hline $\begin{array}{l}\text { P90-P97: Outros transtornos } \\
\text { originados no período perinatal }\end{array}$ & 7 & 10,9 & 6 & 1 & - & - & - & 24 & 36,9 & 24 & 0 & 0 & 0 & - & $242,9^{c}$ \\
\hline $\begin{array}{l}\text { Q00-Q07: Malformações congênitas } \\
\text { do Sistema Nervoso }\end{array}$ & 1 & 1,6 & 1 & - & - & - & - & 1 & 1,5 & 1 & 0 & 0 & 0 & - & 0,0 \\
\hline $\begin{array}{l}\text { Q20-Q28: Malformações congênitas } \\
\text { do Aparelho circulatório }\end{array}$ & - & - & - & - & - & - & - & 1 & 1,5 & 0 & 0 & 1 & 0 & - & - \\
\hline $\begin{array}{l}\text { Q80-Q89: Outras Malformações } \\
\text { congênitas }^{\text {b }}\end{array}$ & 1 & 1,6 & - & 1 & - & - & - & 3 & 4,6 & 2 & 0 & 1 & 0 & - & 200,0 \\
\hline $\begin{array}{l}\text { Q90-Q99: Anomalias crossômicas, } \\
\text { não classificadas em outra parte }\end{array}$ & 1 & 1,6 & - & 0 & 1 & - & - & - & - & - & - & - & - & - & - \\
\hline Total & 64 & 100 & 37 & 19 & 6 & 2 & - & 65 & 100 & 40 & 13 & 8 & 3 & - & - \\
\hline
\end{tabular}

no período perinatal (pesquisa) (Tabela 2). A média de diagnósticos além da CB certificados pelos pesquisadores foi 1,9 e informada pelo SIM, 1,3.

Mais da metade das causas dos óbitos neonatais no nível de agrupamento foram também devidas aos Transtornos respiratórios e cardiovasculares específicos do periodo perinatal (P20-P29) e Fatores maternos e por complicações da gravidez, do trabalho de parto e parto (P00-P004) quando informados no SIM $(54,5 \%)$ e certificados pela pesquisa $(56,8 \%)$, alternando apenas os primeiros e segundos postos de maior frequência. Entre os óbitos neonatais informados pelo SIM, todos apresentaram pelo menos um diagnóstico além $\mathrm{da} \mathrm{CB}$ e em cinco registros este valor ultrapassou quatro. Na pesquisa, 11 óbitos neonatais apresentaram apenas a CB no atestado de óbito e o número máximo de diagnósticos além da $\mathrm{CB}$ foi quatro. Os principais agrupamentos, P20-P29 entre os óbitos neonatais informados pelo SIM e P00-P004 certificados pela pesquisa apresentaram um número maior de diagnósticos além da CB (tabela 2). O número médio de diagnósticos além da $\mathrm{CB}$ dos óbitos neonatais foi superior ao dos natimortos, sendo também o da pesquisa $(3,0)$ superior ao informado pelo $\operatorname{SIM}(2,7)$. 
Tabela 2. continuação

\begin{tabular}{|c|c|c|c|c|c|c|c|c|c|c|c|c|c|c|c|}
\hline \multirow{3}{*}{$\begin{array}{l}\text { Causa Básica }(\mathrm{CB}) \text { por Agrupamentos } \\
\quad \text { (Classificação Estatística } \\
\text { Internacional de Doenças e Problemas } \\
\text { Relacionados à Saúde - 10a Revisão) }\end{array}$} & \multicolumn{7}{|c|}{ SIM } & \multicolumn{7}{|c|}{ Pesquisa } & \multirow{3}{*}{$\begin{array}{c}\text { Mudança } \\
\text { Causa } \\
\text { Básica } \\
\%\end{array}$} \\
\hline & \multirow[t]{2}{*}{$\mathbf{n}^{0}$} & \multirow[t]{2}{*}{$\%$} & \multicolumn{5}{|c|}{$\begin{array}{l}\mathbf{n}^{0} \text { diagnósticos } \\
\text { além da CB }\end{array}$} & \multirow[t]{2}{*}{$\mathbf{n}^{0}$} & \multirow[t]{2}{*}{$\%$} & \multicolumn{5}{|c|}{$\begin{array}{l}\mathbf{n}^{0} \text { diagnóticos } \\
\text { além da CB }\end{array}$} & \\
\hline & & & 0 & 1 & 2 & 3 & $\geq 4$ & & & 0 & 1 & 2 & 3 & $\geq 4$ & \\
\hline \multicolumn{16}{|l|}{ Óbitos Neonatais } \\
\hline $\begin{array}{l}\text { A50-A54: Infecções de transmissão } \\
\text { predominantemente sexual }\end{array}$ & - & - & - & - & - & - & - & 1 & 2,3 & 1 & - & - & - & - & - \\
\hline $\begin{array}{l}\text { P00-P04: Fatores maternos e } \\
\text { complicações (gravidez/trabalho de } \\
\text { parto/parto) }\end{array}$ & 11 & 25 & - & - & 2 & 4 & 5 & 17 & 38,6 & - & 6 & 9 & 1 & 1 & 54,5 \\
\hline $\begin{array}{l}\text { P05-P08: Transtornos relacionados } \\
\text { com duração da gestação e crescimento } \\
\text { fetal }\end{array}$ & 3 & 6,8 & - & 1 & 2 & - & - & 6 & 13,6 & 6 & - & - & - & - & 100,0 \\
\hline P10-P15: Traumatismo de parto & - & - & - & - & - & - & - & 1 & 2,3 & - & - & 1 & - & - & - \\
\hline $\begin{array}{l}\text { P20-P29: Transtornos respiratórios/ } \\
\text { cardiovasculares específicos período } \\
\text { perinatal }\end{array}$ & 13 & 29,5 & - & 1 & 5 & 5 & 2 & 8 & 18,2 & 1 & 2 & 3 & 1 & 1 & $-38,5$ \\
\hline $\begin{array}{l}\text { P35-P39: Infecções específicas do } \\
\text { período perinatal }\end{array}$ & 6 & 13,6 & - & 1 & - & 1 & 4 & 1 & 2,3 & - & 1 & - & - & - & $-83,3$ \\
\hline $\begin{array}{l}\text { P75-P78: Transtornos do aparelho } \\
\text { digestivo do feto e do RN }\end{array}$ & - & - & - & - & - & - & - & 1 & 2,3 & - & 1 & - & - & - & - \\
\hline $\begin{array}{l}\text { P90-P97: Outros transtornos } \\
\text { originados n período perinatal }\end{array}$ & 1 & 2,3 & - & - & - & - & 1 & - & - & - & - & - & - & - & - \\
\hline $\begin{array}{l}\text { Q00-Q07: Malformações congênitas do } \\
\text { Sistema Nervoso }\end{array}$ & 5 & 11,4 & - & 2 & 2 & 1 & - & 5 & 11,4 & 2 & 1 & 1 & 1 & - & 0,0 \\
\hline $\begin{array}{l}\text { Q20-Q28:Malformações congênitas do } \\
\text { Aparelho circulatório }\end{array}$ & 1 & 2,3 & - & - & 1 & - & - & 1 & 2,3 & 1 & - & - & - & - & 0,0 \\
\hline $\begin{array}{l}\text { Q30-Q34: Malformação congênita do } \\
\text { nariz }\end{array}$ & 2 & 4,5 & - & - & 1 & 0 & 1 & 1 & 2,3 & - & - & - & 1 & - & $-50,0$ \\
\hline $\begin{array}{l}\text { Q60-Q64: Malformações congênitas do } \\
\text { Aparelho urinário }\end{array}$ & 1 & 2,3 & - & 1 & - & - & - & 1 & 2,3 & - & - & - & 1 & - & 0,0 \\
\hline $\begin{array}{l}\text { Q65-Q79: Malformações congênitas/ } \\
\text { deformidades aparelho osteomuscular }\end{array}$ & 1 & - & - & - & - & - & 1 & - & - & - & - & - & - & - & - \\
\hline W00-W19: Quedas & - & - & - & - & - & - & - & 1 & 2,3 & - & 1 & - & - & - & - \\
\hline Total & 44 & 100 & - & 6 & 13 & 11 & 14 & 44 & 100 & 11 & 12 & 14 & 5 & 2 & \\
\hline
\end{tabular}

a óbito fetal ( $\geq 22$ semanas de gestação); bUm aborto foi corrigido para natimorto (não foi registrado no Sistema de Informações sobre Mortalidade); ${ }^{\mathrm{C}}$ Teste de Mcnemar $(\mathrm{p}<0,001)$.

Fontes: Sistema de Informações sobre Mortalidade (PRO-AIM São Paulo; Secretaria de Estado de Saúde Rio de Janeiro).

\section{Mudanças da causa básica de morte dos conceptos}

A principal mudança de CB específica dos óbitos fetais foi de hipóxia intrauterina não especificada (P209), informada pelo SIM (30), para mortes fetais de causas não especificadas (P95), certificadas pelos pesquisadores (16). Em todas as situações em que essa modificação ocorreu não havia diagnóstico além da causa básica (SIM e pesquisa). Para oito óbitos fetais cuja CB infor- mada pelo SIM era P209, havia como diagnóstico além da $\mathrm{CB}$, seis no agrupamento Fatores maternos e complicações (P00-P04) e dois Transtornos endócrinos e metabólicos transitórios e específicos (P70-74) que passaram para CB pelos pesquisadores. Para um óbito fetal, a CB hipóxia intrauterina não especificada no SIM mudou para sifilis congênita precoce sintomática (A500) pela pesquisa. Para nenhum óbito fetal foi mantida hipóxia intrauterina como causa básica após certificação pela pesquisa. 
As mudanças de causas específicas dos óbitos neonatais entre SIM e pesquisa foram mais distribuídas entre os códigos da CID. Entretanto, duas mudanças de $\mathrm{CB}$ dos óbitos neonatais registradas no SIM e certificadas na pesquisa chamaram a atenção. Em um óbito, septicemia bacteriana não especificada (P369) foi informada pelo SIM e Sífilis congênita precoce sintomática (A500) certificada pelos pesquisadores. A segunda mudança foi da causa natural prematuridade não especificada (P073) informada pelo SIM para a causa não natural, outras quedas no mesmo nivel (W18), certificada pelos pesquisadores.

Mudanças de CB estatisticamente significantes informadas pelo SIM e pela pesquisa ocorreram apenas por agrupamento (P20-P29 e P90-P97) para os óbitos fetais $(\mathrm{p}<0,01)$ (Tabela 2$)$.

\section{Confiabilidade da causa básica de morte dos conceptos}

A confiabilidade das CB neonatais foi superior à dos óbitos fetais, verificando-se que, à medida que as causas de morte são mais especificadas, diminui a concordância entre o SIM e pesquisa (Tabela 3). A concordância percentual por capítulo da CID foi muito boa tanto para natimortos quanto para óbitos neonatais; o coeficiente kappa apresentou concordância substancial $(0,70)$ e moderada $(0,54)$ para os óbitos neonatais e natimortos, respectivamente. Os óbitos neonatais apresentam concordância moderada $(k a p p a=0,40)$ e razoável no nível de três caracteres (kappa $=0,23)$ e quatro caracteres (kappa $=0,25)$. Para os óbitos fetais, a concordância por agrupamento e causa específica foi superficial (Tabela 3).

\section{Discussão}

Este estudo permitiu avaliar todo o espectro das mortes dependentes da ocorrência de gravidez.

Ocorreu um óbito materno para $7.335 \mathrm{NV}$ nesta pesquisa. Em número absoluto, óbito materno é um evento raro e possivelmente a ocorrência no centro SP se deveu mais à frequência de $\mathrm{NV}(75,8 \%)$ do que à demanda de gravidez de risco e/ou uma pior assistência obstétrica. No estudo seccional multicêntrico em 2010/11, em sete maternidades brasileiras participantes ocorreu um óbito materno e a razão de mortalidade materna foi $14,2 / 100.000 \mathrm{NV}^{21}$, valor próximo ao deste estudo.

O pior cenário da mortalidade fetal e neonatal foi no centro RJ. Exceto uma maternidade paulistana, as demais são referência para gravidez de risco. Deve-se considerar que além de possíveis diferenças na qualidade da assistência obstétrica e neonatal entre os centros RJ e SP, na maternidade da cidade de Niterói, referência para a região metropolitana II do Estado do Rio de Janeiro, grande parcela das parturientes assistidas é de risco e oriundas de municípios mais empobrecidos, elevando os riscos de morte de mulheres e conceptos. O risco de mortalidade fetal estimado para este estudo priorizou o peso ao nascer seguindo a definição da $\mathrm{CID}^{10}$ e, portanto, pode estar subestimado ${ }^{27}$. Esta subestimação pode ser maior quando a frequência de nascimentos com restrição do crescimento intrauterino é alta ${ }^{27}$.

As características sociodemográficas da parturiente que evoluiu para morte materna neste estudo, além da causa de morte associada (tuberculose pulmonar), expressam condições de vulnerabilidade ${ }^{2}$. As causas de morte certificadas,

Tabela 3. Concordância percentual entre as causas de óbitos fetais e neonatais informadas pelo Sistema de Informações sobre Mortalidade e certificadas pela pesquisa em maternidades públicas das cidades Rio de Janeiro, Niterói e São Paulo, 2011.

\begin{tabular}{|c|c|c|c|c|c|c|c|c|}
\hline \multirow[b]{3}{*}{ Capítulo } & \multicolumn{4}{|c|}{ Óbitos fetais ${ }^{a}(n=64)$} & \multicolumn{4}{|c|}{ Óbitos neonatais $(\mathrm{n}=44)$} \\
\hline & \multirow{2}{*}{$\begin{array}{c}\begin{array}{c}\text { concordância } \\
\%\end{array} \\
90,63\end{array}$} & \multirow{2}{*}{$\begin{array}{c}\begin{array}{c}\text { Coef. } \\
\text { kappa }^{\text {b }}\end{array} \\
0,54\end{array}$} & \multicolumn{2}{|c|}{ IC 95\% } & \multirow{2}{*}{ 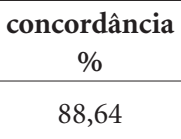 } & \multirow{2}{*}{$\begin{array}{c}\begin{array}{c}\text { Coef. } \\
\text { kappa }^{\mathrm{b}}\end{array} \\
0,70\end{array}$} & \multicolumn{2}{|c|}{ IC 95\% } \\
\hline & & & 0,34 & 0,78 & & & - & - \\
\hline Agrupamento & 31,25 & 0,16 & 0,06 & 0,26 & 51,16 & 0,40 & 0,24 & 0,62 \\
\hline Categorias de 3 caracteres & 26,56 & 0,17 & 0,13 & 0,21 & 27,27 & 0,23 & 0,10 & 0,27 \\
\hline Categorias de 4 caracteres & 20,31 & 0,14 & 0,12 & 0,21 & 27,7 & 0,25 & 0,18 & 0,29 \\
\hline
\end{tabular}

IC 95\%: Intervalo de Confiança de 95\% do coeficiente kappa. ${ }^{a}$ Óbitos fetais correspondem aos óbitos fetais do componente perinatal (idade gestacional $\geq 22$ semanas). ${ }^{\text {pp}}$-valor $<0,001$.

Fontes: Sistema de Informações sobre Mortalidade (PRO-AIM, São Paulo e Secretaria de Estado de Saúde do Rio de Janeiro). 
hemorragia e obstrução do parto, são as complicações mais frequentes no momento do nascimento e comuns à morte materna, neonatal e fe$\mathrm{tal}^{22}$. Esta tragédia resultou em duas mortes, mãe e concepto, e quatro órfãos. Apenas as melhorias da atenção profissional e institucional ao parto, independentemente das condições das mulheres, são capazes de reduzir significativamente a ocorrência de mortes maternas ${ }^{3}$.

Como apontado em outros estudos ${ }^{8,9,20}$, erros de classificação do tipo de aborto, devido à ilegalidade, resultaram na subestimação de abortos provocados, o que comprometeu o conhecimento sobre o tipo de aborto. No centro RJ, a proporção de abortos provocados foi maior do que em SP, resultado compatível com o inquérito nacional ${ }^{20}$. No presente estudo, entre os abortos provocados, o uso de misoprostol foi relatado por quase a totalidade das respondentes, apesar do medicamento ser restrito a hospitais credenciados. O comércio ilegal do misoprostol favorece sua adulteração e o risco de venda de apresentações com subdoses ou sem o princípio ativo compromete sua eficácia ${ }^{28}$, expondo as mulheres a situações de risco, quer seja pelo acesso à medicação ou pelo êxito não obtido ${ }^{29}$. Aborto é uma causa importante de morbi-mortalidade materna, principalmente quando realizado em condições inseguras ${ }^{30}$.

Aborto, óbito fetal, óbito neonatal e sobrevivente infantil apresentaram-se como grupos distintos corroborando resultados de estudos sobre fatores de risco e mortalidade ${ }^{11-13,15,20}$, exceto por algumas peculiaridades. Conceptos mostraramse homogêneos em relação às variáveis nascidos mortos anteriores e uso de tabaco ( $\mathrm{p}$ valor $>0,20$ ). A natimortalidade prévia foi descrita como um fator de risco independente para óbitos fetais ${ }^{15}$ e óbitos neonatais ${ }^{13}$ e, não apenas o tabagismo, mas também o uso de álcool e drogas, não estiveram associados aos óbitos fetais ${ }^{15}$. A baixa idade gestacional dos abortos pode ter influenciado o acesso ao pré-natal.

Destacaram-se pela alta frequência, algumas características sociodemográficas como adolescência entre os óbitos fetais, cor de pele materna preta entre mortes fetais e neonatais ${ }^{13}$ e ausência de companheiro nos casos de aborto ou óbito fe$\mathrm{tal}^{15}$. Essas características sugerem a influência de desigualdades sociais na assistência e nos desfechos de saúde ${ }^{2}$.

Entre os óbitos perinatais predominaram óbitos fetais $(66,3 \%)$; óbitos neonatais ocorreram principalmente nas primeiras 48 horas, em concordância com a literatura ${ }^{1,6,13}$.
Apesar da obrigatoriedade ${ }^{31}$, um pouco mais da metade dos óbitos fetais e neonatais havia sido investigada. A investigação do óbito possibilita qualificar a certificação das causas de morte. Em 2011, a investigação não era universal e as secretarias de saúde estabeleciam critérios de prioridade ${ }^{32,33}$. Da mesma forma, espera-se que a certificação das causas de morte pelos pesquisadores seja mais qualificada do que a certificação original na maioria dos casos. As diferenças entre as certificações do SIM e da pesquisa repercutiram na ordenação de postos das CB e na concordância, fundamentalmente no nível de agrupamento da CID. Contudo, a subestimação de sífilis congênita (capítulo I) entre natimortos e óbitos neonatais e queda (capítulo XX) nos óbitos neonatais pelo SIM contribuíram para enfraquecer a concordância também no nível de capítulo.

Discordâncias de CB informadas pelo SIM e pela pesquisa foram maiores entre os óbitos fetais comparados aos óbitos neonatais e aumentam com a especificidade da causa (de capítulo a quatro caracteres). Evidencia-se por um lado o despreparo dos profissionais em reconhecer a cadeia causal que resultou na morte ${ }^{18} \mathrm{e}$, por outro, a presença de um complexo inter-relacionamento entre as diversas causas ${ }^{18}$. Estudos nacionais mostraram baixa confiabilidade da CB de óbitos fetais, perinatais e infantis ${ }^{15,17,18,34,35}$.

Entre os óbitos fetais, a principal modificação de causa básica entre o SIM e pesquisa ocorreu de hipóxia intrauterina não especificada para causas de morte fetal não especificada. Esta mudança também foi destacada nos estudos similares de óbitos fetais ${ }^{15}$ e perinatais ${ }^{35}$ e pode refletir a incompletude do prontuário do óbito fetal, a não realização de necropsia ou, a falta de informações nos laudos ${ }^{17,35}$. A ausência de informações clínicas da gestação e do parto e a não inclusão das placentas na necropsia dificultam a precisão das causas de morte ${ }^{17}$.

Hipóxia intrauterina representa o estágio terminal da cadeia causal da mortalidade, portanto, não deve ser certificada como CB; e causas de morte fetal não especificada são as "causas mal definidas" dos óbitos perinatais. Ambos os diagnósticos (P209 e P95) são inespecíficos para direcionar medidas de prevenção ${ }^{36}$.

Apesar do pequeno número absoluto de óbitos fetais, as frequências de sífilis congênita (A50-A54) e de diabetes (P70-P74) foram, respectivamente, 2,5 e cinco vezes maiores quando comparadas à informação do SIM. Essas causas de mortalidade fetal são decorrentes de morbidades maternas consideradas modificáveis pela 
melhor assistência obstétrica e frequentes em países de média e baixa renda ${ }^{11}$. No Brasil, a prevalência de sífilis na gestação é maior na ausência de pré-natal e, mesmo quando realizado, o início tardio pode ser uma barreira para diagnóstico e tratamento na idade gestacional recomendada ${ }^{37}$. A fração atribuível populacional mundial da sífilis e da diabetes pré-gestacional na mortalidade fetal foi estimada em cerca de $8 \%$ e $7 \%$, respectivamente ${ }^{11}$. Sífilis congênita também foi subregistrada como causa básica entre os óbitos neonatais em concordância com estudo de Alagoas na década anterior ${ }^{18}$. Os autores destacam a evitabilidade de mortes neonatais por sífilis congênita e diabetes na gestação, entre outras causas.

A qualificação da CB dos óbitos fetais e neonatais pela pesquisa pode ser avaliada também pelo aumento do número de diagnósticos certificados. Por vezes, os médicos deixam de informar diagnósticos além da CB no atestado de óbito por já estarem registrados no prontuário ${ }^{38}$. Particularmente para os óbitos fetais, questões referentes aos códigos da CID, escassos e incompletos para as condições placentárias, e ao próprio modelo da Declaração de Óbito que não contempla informações sobre intercorrências da gravidez e do parto devem ser consideradas ${ }^{27}$.

Uma limitação da pesquisa foi a inexistência de um Serviço de Verificação do Óbito nas cidades do Estado do Rio de Janeiro e, no caso

\section{Colaboradores}

PL Kale, MHP Mello Jorge, SC Fonseca, AM Cascão, KS Silva, AC Reis e MT Taniguchi são responsáveis pelo conteúdo do artigo e concordam com a versão enviada para publicação. da cidade de São Paulo que dispõe do serviço, alguns resultados foram imprecisos para qualificar a causa de morte, como sinalizado por Almeida et al. ${ }^{17}$. Adicionalmente, a estratégia de captação dos recém-nascidos em maternidades com maior número de nascimentos no SUS nas cidades integrantes da pesquisa, semelhante à estratégia amostral utilizada no inquérito multicêntrico da Saúde Materna e do Recém-nascido da Organização Mundial de Saúde ${ }^{39}$, não correspondeu a uma amostra aleatória.

Como pontos positivos do estudo ressalta-se a inexistência praticamente de erro de classificação entre as perdas fetais (aborto e óbitos fetais) e dos óbitos fetais com os nascidos vivos, estas últimas já sinalizadas em publicação com a base de dados do inquérito na cidade ${ }^{40}$ de São Paulo.

Para o melhor preenchimento da Declaração de Óbito e consequente precisão das causas de morte é necessário enfatizar aos médicos e principalmente aos estudantes de Medicina, as definições das mortes (materna, aborto, componente fetal do óbito perinatal e infantil por componentes) e a inclusão de todas as doenças nos atestados de óbitos ${ }^{38}$, as do recém-nascido e aquelas consequentes às morbidades maternas, impactando positivamente na qualidade da assistência.

A assistência de boa qualidade no planejamento reprodutivo, pré-natal, durante o parto e nascimento resultará na prevenção de abortos, mortes fetal, materna e neonatal ${ }^{11}$. 


\section{Referências}

1. World Health Organization (WHO). Global Strategy for Women's and Children's health. Geneva: WHO; 2010. [acessado 2016 Jun 27]. Disponível em: http:// www.who.int/pmnch/topics/maternal/20100914_gswch_en.pdf?ua=1

2. United Nations Development Programme (UNDP). A social determinants approach to maternal health. [acessado 2016 Jan 14]. Disponível em: http://www.undp.org/ content/dam/undp/library/Democratic\%20Governance/Discussion\%20Paper\%20MaternalHealth.pdf

3. Cecatt, JG. Saúde da mulher: enfoque da evidência científica para a prevenção da morbidade e mortalidade materna. Rev Bras Saude Mater Infant 2005; 5(1):911.

4. Brasil. Ministério da Saúde (MS). Secretaria de Vigilância em Saúde. DATASUS. [acessado 2016 Jan 5]. Disponível em: http://www2.datasus.gov.br/DATASUS/ index.php?area $=0205$

5. World Health Organization (WHO). Trends in maternal mortality: 1990 to 2015. Estimates by WHO, UNICEF, INFPA, The World Bank and the United Nations Population Division. Geneva: WHO; 2015.

6. Adams MM, Alexander GR, Kirby RS, Wingate MS. The continuum of Reproductive Loss from pregnancy through infancy. In: Adams MM, Alexander GR, Kirby RS, Wingate MS. Perinatal Epidemiology for Public Health. São Paulo: Spring Ed.; 2009. p. 155-224.

7. Sedgh G, Henshaw S, Singh S, Ahman E, Shah IH. Induced 2. abortion: estimated rates and trends worldwide. Lancet 2007; 370(9595):1338-1345.

8. Diniz D, Medeiros A. Aborto no Brasil: uma pesquisa domiciliar com técnica de urna. Cien Saude Colet 2010; 15(Supl. 1):959-966.

9. Monteiro MFG, Adesse L, Drezett J. Atualização das estimativas da magnitude do aborto induzido, taxas por mil mulheres e razões por 100 nascimentos vivos do aborto induzido por faixa etária e grandes regiões. Brasil, 1995 a 2013. Reprod \& Clim 2015; 30(1):11-18.

10. Organização Mundial de Saúde (OMS). Classificação Estatística Internacional de Doenças e Problemas Relacionados à Saúde - 10a Revisão. São Paulo: CBCD/ EDUSP; 1995. v I e II.

11. Lawn JE, Blencowe H, Waiswa P, Amouzo A, Mathers C, Hogan D, Flenady V, Frøen JF,Qureshi ZU, Calderwood C, Shiekh S, Jassi FB, You D, McClure M, Matha Mi, Cousens S, for The Lancet Ending Preventable Stillbirths Series study group* with The Lancet Stillbirth Epidemiology investigator group. Stillbirths: rates, risk factors, and acceleration towards 2030. Lancet 2016; 18 .

12. McClure EM, Pasha O, Goudar SS, Chomba E, Garces A, Tshefu A, Althabe F, Esamai F, Patel A, Wright LL, Moore J, Kodkany BS, Belizan JM, Saleem S, Derman RJ, Carlo WA, Hambidge KM, Buekens P, Liechty EA, Bose C, Koso-Thomas M, Jobe AH, Goldenberg RL; Global Network Investigators. Epidemiology of stillbirth in low-middle income countries: a Global Network Study. Acta Obstet Gynecol Scand 2011; 90(12):1379-1385.

13. Lansky S, Friche AAL, Silva AAM, Campos D, Bittencourt SDA, Carvalho ML, frias PG, Cavalcante RS, Cunha AAL. Pesquisa Nascer no Brasil: perfil da mortalidade neonatal e avaliação da assistência à gestante e ao recém-nascido. Cad Saude Publica 2014; 30 (Supl. 1):S192-S207.
14. Lee EJ, Gambatese M, Begier E, Soto A, Das T, Madsen A. Understanding Perinatal Death: A Systematic Analysis of New York City Fetal and Neonatal Death Vital Record Data and Implications for Improvement, 20072011. Matern Child Health J 2014; 18(8):1945-1954.

15. Barbeiro FMS, Fonseca SC, Tauffer MG, Ferreira MSS, Silva FP, Ventura PM, Quadros JI. Óbitos fetais no Brasil: revisão sistemática. Rev Saude Publica 2015; 49:22.

16. Barbuscia DM, Rodrigues-Júnior AL. Completude da informação nas declarações de nascido vivo e nas declarações de óbito, neonatal precoce e fetal, da região de Ribeirão Preto, São Paulo, Brasil, 2000-2007. Cad Saude Publica 2011; 27(6):1192-1200.

17. Almeida MF, Alencar GP, Schoeps D, Minuci EG, Silva ZP, Ortiz LP, Novaes HMD, Alencar AP, Raspatini PR, Santos PC. Qualidade das informações registradas nas declarações de óbito fetal em São Paulo, SP. Rev Saude Publica 2011; 45(5):845-53.

18. Pedrosa LCDO, Sarinho SW, Ordonha MR. Análise da qualidade da informação sobre causa básica de óbitos neonatais registrados no Sistema de Informações sobre Mortalidade: um estudo para Maceió, Alagoas, Brasil, 2001-2002. Cad Saude Publica, 2007; 23(10):23852395.

19. Lansky S, França E, Leal MC. Mortalidade perinatal e evitabilidade: revisão da literatura. Rev Saude Publica 2002; 36(6):759-772.

20. Cecatti JG, Guerra CVQL, Sousa MH, Menezes GMS. Aborto no Brasil: um enfoque demográfico. Rev Bras Ginecol Obstet 2010; 32(3):105-111.

21. Chaves SC, Cecatti JG, Carroli G, Lumbiganon P, Hogue CJ, Mori R, Zhang J, Jayaratne K, Togoobaatar G, Pileggi-Castro C, Bohren M, Vogel JP, Tunçalp Ö, Oladapo OT, Gülmezoglu AM, Temmerman M, Souza JP. Obstetric transition in the World Health Organization Multicountry Survey on Maternal and Newborn Health: exploring pathways for maternal mortality reduction. Rev Panam Salud Publica 2015; 37(4/5):203210.

22. Goldemberg RL, MacClure EM, Bhutta ZGA, Belizán JM, Reddy UM, Rubens GE, Mabeya H, Flenady V, Darmstadt GL; for The Lancet's Stillbirths Series steering committee. Stillbirths: the vision for 2020. Lancet 2011; 377(9779):1798-1805.

23. Camargo Júnior KR, Coeli CM. RecLink 3: nova versão do programa que implementa a técnica de associação probabilística de registros (probabilistic record linkage). Cad Saúde Coletiva 2006; 14(2):399-404.

24. Rio de Janeiro (Município). Secretaria de Saúde e Defesa Civil do Município do Rio de Janeiro. Roteiro para crítica dos sistemas de informação SINASC e SIM. Rio de Janeiro: [s.n.]; 2008.

25. Mello Jorge MHP, Laurenti R, Gotlieb SLD. Avaliação dos Sistemas de Informações em Saúde no Brasil. Cad Saúde Coletiva 2010; 18(1):7-18.

26. Szklo M, Nieto F. Epidemiology, Beyond the Basics. $2^{\text {nd }}$ ed. Boston: Jones and Barllett Publishers, 2007.

27. Froen JF, Gordijn SJ, Abdel-Aleem H, Bergsjo P, Betran A, Duke CW, Fauveau V, Flenady V, Hinderaker SG, Hofmeyr GJ, Jokhio AH, Lawn J, Lumbiganon P, Merialdi M, Pattinson R, Shankar A. Making stillbirths count, making numbers talk - Issues in data collection for stillbirths. BMC Pregnancy and Childbirth 2009; 9:58. 
28. Diniz D, Medeiros M. Cytotec e Aborto: a polícia, os vendedores e as mulheres. Cien Saude Colet 2012; 17(7):1795-1804.

29. Arilha MM. Misoprostol: percursos, mediações e redes sociais para o acesso ao aborto medicamentoso em contextos de ilegalidade no Estado de São Paulo. Cien Saude Colet 2012; 17(7):1785-1794.

30. Domingos SRF, Merigui MAB. O aborto como causa de morte materna: um pensar para o cuidado da enfermagem. Esc Anna Nery Rev Enferm 2010; 14 (1):177-181.

31. Brasil, Diário Oficial da União. № 10 , sexta-feira, 15 de janeiro de 2010. [acessado 2016 Jan 10]. Disponível em: http://pesquisa.in.gov.br/imprensa/jsp/visualiza/index. jsp?jornal $=1$ \&pagina $=67 \&$ data $=15 / 01 / 2010$

32. Rio de Janeiro. Diário Oficial do Município do Rio de Janeiro. Secretaria de Saúde e Defesa Civil do Rio de Janeiro. Resolução SMS n ${ }^{\circ} 1.257$ de 12 de fevereiro de 2007. [acessado 2015 Dez 16]. Disponível em: http:// doweb.rio.rj.gov.br/ler_pdf.php?edi_id=902\&page $=19$

33. São Paulo. Diário Oficial do Estado de São Paulo. No 37 - DOE de 24/02/11 -Seção 1 - p.34. [acessado 2015 Dez 16]. Disponível em: ftp.saude.sp.gov.br/ftpsessp/ bibliote/informe_eletronico/2011/iels.fev.11/Iels37/E DL-CIB-5_230211.pdf

34. Santos SPC, Lansky S, Ishitani LH, França EB. Óbitos infantis evitáveis em Belo Horizonte: análise de concordância da causa básica, 2010-2011. Rev Bras Saúde Matern Infant 2015; 15(4):389-399.

35. Lansky S, França E, Ishitani L, Xavier CC. Confiabilidade da informação sobre óbito perinatal em Belo Horizonte, 1999: causas de óbito e variáveis selecionadas. Cad Saúde Colet 2010, 18(1):63-70.

36. Assis HM, Siviero PCL, Drumond EF, Machado CJ. Óbitos fetais sob o prisma da evitabilidade: análise preliminar de um estudo para o município de Belo Horizonte Cad Saúde Colet 2014, Rio de Janeiro, 22(3):314317.

37. Domingues RMSM, Szwarcwald CL, Souza Junior PRB, Leal MC. Prevalência de sífilis na gestação e testagem pré-natal: Estudo Nascer no Brasil. Rev Saude Publica 2014; 48(5):766-774.

38. Laurenti R. A análise da mortalidade por causas múltiplas. Rev Saude Publica 1974: 8:421-435.

39. Souza JP, Cecatti JG, Faundes A, Morais SS, Villar J, Carroli G, Gulmezoglu M, Wojdyla D, Zavaleta N, Donner A, Velazco A, Bataglia V, Valladares E, Kublickas M, Acosta A; WHO 2005 global survey on maternal and perinatal health research group. Maternal near miss and maternal death in the 2005 WHO global survey on maternal and perinatal health. Bull World Health Organ 2010; 88(2):113-119.

40. Laurenti R, Mello Jorge MHP, Gotlieb SLD, Oliveira BC, Pimentel EC. O estudo do binômio mãe-filho: descrição e resultados gerais. Rev Bras Epidemiol 2015; 18(2):398-412.

Artigo apresentado em 15/03/2016

Aprovado em 13/07/2016

Versão final apresentada em 15/07/2016 